Reprod. Nutr. Dévelop., 1987, 27 (1 A), 95-108.

\title{
Répercussion de la durée d'éclairement journalier sur l'évolution des cellules nourricières et de la lignée mâle dans l'ovotestis d'Helix aspersa
}

\author{
P. GOMOT, Bernadette GRIFFOND
}

Laboratoire de Zoologie et Embryologie,

UA C.N.R.S., $n^{\circ}$ 040687, Faculté des Sciences et des Techniques, place Maréchal Leclerc, 25030 Besançon Cedex, France.

Summary. The effect of lighting duration on the evolution of nurse cells and male line in the Helix aspersa ovotestis.

Adult snails (Helix aspersa) were bred under two photoperiods i.e. long-day: $18 \mathrm{~h}$ (JL), short-day : $8 \mathrm{~h}$ (JC) immediately after a 4 month hibernation. In these conditions only the long-day snails began to lay eggs from the 10th week of experiment, whereas the others did not.

To explain these differences, the structure and functioning of the hermaphroditic glands were investigated in both groups, 6 and 10 weeks later, using electron microscopic and quantitative biochemical methods.

At 6 weeks, compared to short-day cycles $(\mathrm{JC})$, long-days (JL) highly increased spermatogonial multiplication measured by ${ }^{3} \mathrm{H}$ thymidine incorporation and on the other hand shortened the duration of spermatogenesis. Ultrastructural observations showed that modifications of the nurse cells were strongly correlated with the evolution of the male line.

At 10 weeks, the rates of spermatogonial multiplication were rather similar in all snails.

In spite of the absence of anomalies in the male cells, no reproduction occurred among the short-day animals ; this is probably due to a low concentration of sperm, suggesting an insufficient stimulation by the neuroendocrine controlling centers.

\section{Introduction.}

L'analyse expérimentale de l'action des facteurs externes sur la gamétogenèse et la reproduction des Gastéropodes n'a été abordée que récemment (Tardy, 1982). En particulier, les données concernant les effets de la photopériode sont relativement peu nombreuses. Chez les Limaces, Henderson et Pelluet (1960) montrent qu'un éclairement continu stimule la spermiogenèse de Deroceras reticulatum ; la photopériode est un facteur d'environnement important dans le contrôle de la différenciation du tractus génital (Sokolove et McCrone, 1978) et de la synthèse d'ADN gonadique chez Limax maximus (Sokolove et al., 1983). Chez Lymnaea stagnalis, les longues photophases stimulent la production d'œufs (Bohlken et Joosse, 1982) et accélèrent la maturation sexuelle (Joosse, 1984). Chez l'Escargot Helix aspersa, elles favorisent la reproduction (Enée et al., 1982), tandis que les courtes photophases l'inhibent plus ou moins totalement (Stephens et Stephens, 1966 ; Enée et al., 1982). Cependant, contrairement à ce qui se passe chez des Escargots maintenus en inactivité à basse température 
(Gomot et al., 1986), la spermatogenèse n'est pas bloquée chez des animaux élevés en jours courts (Gomot et Gomot, 1985). Les raisons de la faible proportion d'accouplements et de pontes observée dans ces conditions restant à élucider, nous avons réalisé d'une part une étude histologique et ultrastructurale de la gonade, visant à rechercher l'existence d'éventuelles anomalies au niveau des cellules germinales ou accessoires, d'autre part une étude biochimique et autoradiographique permettant d'évaluer le taux de multiplication des cellules gonadiques. Le présent travail a donc pour objet l'analyse des répercussions de deux photophases, une courte $(\mathrm{JC}$ ) et une longue (JL) sur les aspects qualitatifs et quantitatifs du fonctionnement de l'ovotestis d'animaux adultes, au cours de la période qui sépare la sortie de l'hibernation de l'entrée en reproduction.

\section{Matériel et méthodes.}

\section{Les animaux.}

Des Escargots adultes "bordés ", recueillis enterrés dans la nature au début de leur période de repos hivernal en octobre, sont conservés en chambre froide obscure à $7 \pm 1^{\circ} \mathrm{C}$ pendant 4 mois au Centre Universitaire d'Héliciculture $\left({ }^{1}\right)$. On réalise alors 4 lots de 30 animaux qui sont placés en atmosphère favorable à une reprise d'activité : hygrométrie constante de $95 \%$ et alternance de température de $17 \pm 0,2{ }^{\circ} \mathrm{C}$ pendant $12 \mathrm{~h}(6 \mathrm{~h}$ à $18 \mathrm{~h})$ et de $12 \pm 0,2^{\circ} \mathrm{C}$ pendant le reste de la journée, mimant ainsi la succession naturelle de température entre le jour et la nuit. En ce qui concerne l'éclairement, les animaux sont partagés en deux groupes, l'un soumis à une photophase de $18 \mathrm{~h}(\mathrm{JL})$ et l'autre à une photophase de $8 \mathrm{~h}(\mathrm{JC})$ par $24 \mathrm{~h}$.

Au cours de l'expérience, le nombre d'accouplements est noté chaque jour et les pontes sont relevées chaque semaine. L'expression de ce comportement de reproduction a été exposée précédemment (Gomot et Gomot, 1985).

\section{Méthodes.}

Prélèvements d'organes. - Des expériences antérieures, réalisées dans les mêmes conditions d'éclairement et de température (Bonnefoy-Claudet et Deray, 1984), ayant démontré qu'après une hibernation de 6 mois on n'observe pratiquement pas d'accouplements avant le $40^{\mathrm{e}}$ jour, nous avons choisi d'étudier les gonades au $39^{\circ}$ (début des accouplements) et au 69ª jour de l'expérience (début de la ponte).

A la $6^{\mathrm{e}}$ et à la $10^{\mathrm{e}}$ semaine de l'expérience [prélèvements $\mathrm{P} 1$ (39e jour) et P2 $\left(69^{\mathrm{e}}\right.$ jour)], 5 animaux de chaque groupe reçoivent 3 injections de thymidine méthyle ${ }^{3} \mathrm{H}\left({ }^{2}\right)$ à la dose de $5 \mu \mathrm{Ci}$ par gramme de poids frais dans la cavité générale à $2 \mathrm{~h}$ d'intervalle à partir de $9 \mathrm{~h}$ (soit $1 \mathrm{~h}$ après le début de la période d'éclairement journalier). Les animaux sont sacrifiés $5 \mathrm{~h}$ après la première injection. La gonade, prélevée rapidement, est pesée afin de déterminer l'indice gonadique

(1) Centre Universitaire d'Héliciculture - 5, rue Ronchaux - 25000 Besançon.

(2) C.E.A. (Commissariat à I'Energie Atomique) - 91191 Gif-sur-Yvette. 
apprécié par le rapport : poids de la gonade/poids de l'Escargot $\times 10^{5}$. Elle est ensuite divisée en trois parties :

- la première est fixée et incluse suivant les conditions décrites par Courtot et Gomot (1982) pour la réalisation de coupes semi-fines nécessaires à l'étude autoradiographique et de coupes ultrafines destinées à l'observation titrastructurale ; - quant aux deux autres fragments, l'un est congelé aussitôt à $-30^{\circ} \mathrm{C}$ pour le dosage des protéines et l'autre est traité immédiatement pour l'extraction de I'ADN afin d'estimer l'incorporation de thymidine marquée dans la gonade.

Histologie et cytologie. - Des coupes semi-fines sont colorées au bleu de toluidine (Trump et al., 1961) tandis que des coupes voisines sont recouvertes d'émulsion llford $L 4$, révélées à l'aide du révélateur D19 et fixées à l'hyposulfite de sodium à $30 \%$. Cette mise en évidence autoradiographique des cellules incorporant la thymidine tritiée est suivie de l'évaluation des cellules marquées par unité de surface gonadique. L'examen ultrastructural est effectué sur coupes ultrafines contrastées selon la méthode de Reynolds (1963).

Biochimie. - L'évaluation de l'incorporation de thymidine tritiée est déterminée par comptage en scintillation liquide de la fraction soluble après hydrolyse à chaud par le PCA (acide perchlorique) 0,5 M selon la méthode employée par Sokolove et al., (1983). La radioactivité est rapportée à la quantité de protéines dosées selon la méthode de Lowry et al., (1951).

Les mesures de radioactivité et les comptages de cellules marquées sont comparés à l'aide du test non paramétrique $U$ de Mann et Whitney (d'après Siegel, 1956).

\section{Résultats.}

\section{Etude histologique et ultrastructurale.}

L'ovotestis d'Helix aspersa adulte est une glande en grappe, constituée de tubules ou acini dans lesquels se distinguent nettement deux régions : une région proximale ou col, limitée par un épithélium composé de cellules plus ou moins cubiques, et une région distale, tapissée par des cellules non germinales dont l'aspect varie selon le stade d'avancement de la gamétogenèse. Les cellules germinales femelles subissent la totalité de leur différenciation contre la paroi à l'intérieur d'une enveloppe folliculaire, tandis que les cellules mâles, en relation avec les cellules nourricières, évoluent dans la lumière des acini. Après 4 mois d'hibernation, les tubules gonadiques sont peu remplis ; ils contiennent principalement des spermatogonies et des spermatocytes mais aussi quelques paquets de spermatozoïdes désordonnés parmi des débris cellulaires. Aucune spermatide n'est observée suggérant un arrêt total de la spermiogenèse. La lignée femelle est représentée par de nombreux ovocytes en préméiose et prévitellogenèse et par quelques gros ovocytes en dégénérescence. 
1) Prélèvement $P 1$.

a) Animaux placés en $J L$. - La lumière des tubules est remplie de cellules mâles à tous les stades de la spermatogenèse, les stades les plus avancés, spermatides et spermatozoïdes, prédominant (fig. 1). Contre les parois, des ovocytes de toutes tailles sont visibles.

Au microscope électronique, la lame basale est bien étalée (fig. 4). Les cellules du col (fig. 2), ancrées les unes aux autres par des jonctions septées et des desmosomes apicaux, possèdent un noyau à contour irrégulier, renfermant quelques blocs d'hétérochromatine et un ou deux nucléoles en position centrale ou pariétale. Leur cytoplasme est garni de rosettes de glycogène souvent associé à des globules lipidiques (fig. 3). L'apex est orné de nombreuses villosités plus ou moins dilatées (fig. 2). Dans la zone distale, les cellules nourricières sont allongées contre la lame basale (fig. 4). Latéralement, elles sont unies par des jonctions septées. Leur noyau vraisemblablement polyploïde, possède un nucléole réticulé et une hétérochromatine dispersée en petits amas. Dans le cytoplasme encombré de lipides et de glycogène, quelques lysosomes sont observés ainsi qu'un appareil de Golgi bien développé. Ces cellules, qui ont souvent une forme pyramidale, émettent vers la lumière des prolongements auxquels sont accrochés des faisceaux de spermatides rondes (fig. 4) ou allongées (fig. 5). II n'est pas rare qu'entre la lame basale et l'assise de cellules nourricières s'intercale une couche, plus ou moins continue, de cellules pariétales très aplaties (fig. 4).

b) Animaux maintenus en $J C$. - L'aspect des acini diffère nettement de celui décrit précédemment. Les tubules, entourés d'un tissu vésiculeux très développé, sont beaucoup moins remplis. Les cellules mâles sont aux stades précoces de la spermatogenèse : spermatogonies et spermatocytes (fig. 6). Chez 2 animaux sur 5 , on peut cependant noter la présence de quelques spermatides et spermatozoïdes. Ainsi la spermiogenèse redémarre seulement. Toutes les étapes de l'ovogenèse (Griffond et Bolzoni-Sungur, 1986) sont représentées.

FIG. 1. - Aspect d'un acinus. La lignée mâle est fortement stimulée $(\times 300)$.

FIG. 2. - Cellules du col d'un acinus. Le noyau est relativement pauvre en hétérochromatine. On notera l'abondance des villosités apicales $(\times 6300)$.

FIG. 3. - Détail du cytoplasme de cellules du col: le glycogène et les lipides sont abondants $(\times 8000)$.

FIG. 4. - Relation typique entre cellule nourricière et spermatides rondes en cours de différenciation $(\times 1900)$.

FIG. 5. - Etroite liaison entre cellule nourricière et faisceau de spermatides allongées $(\times 5000)$.

$c$ : centriole ; $\mathrm{cn}$ : cellule nourricière ; $\mathrm{cp}:$ cellule pariétale ; da : desmosome apical ; go : appareil de Golgi ; $\mathbf{g}$ : tissu glial ; gl : glycogène ; js : jonction septée ; L : lumière ; I : lipides ; lb : lame basale ; $\mathrm{m}$ : mitochondries; $\mathrm{N}$ : noyau ; pi : pièce intermédiaire ; pn : prolongement de cellule nourricière ; sc : spermatocytes ; st : spermatides ; $s z$ : spermatozoïdes ; $t$ : tête de spermatide ; flèches : villosités. 

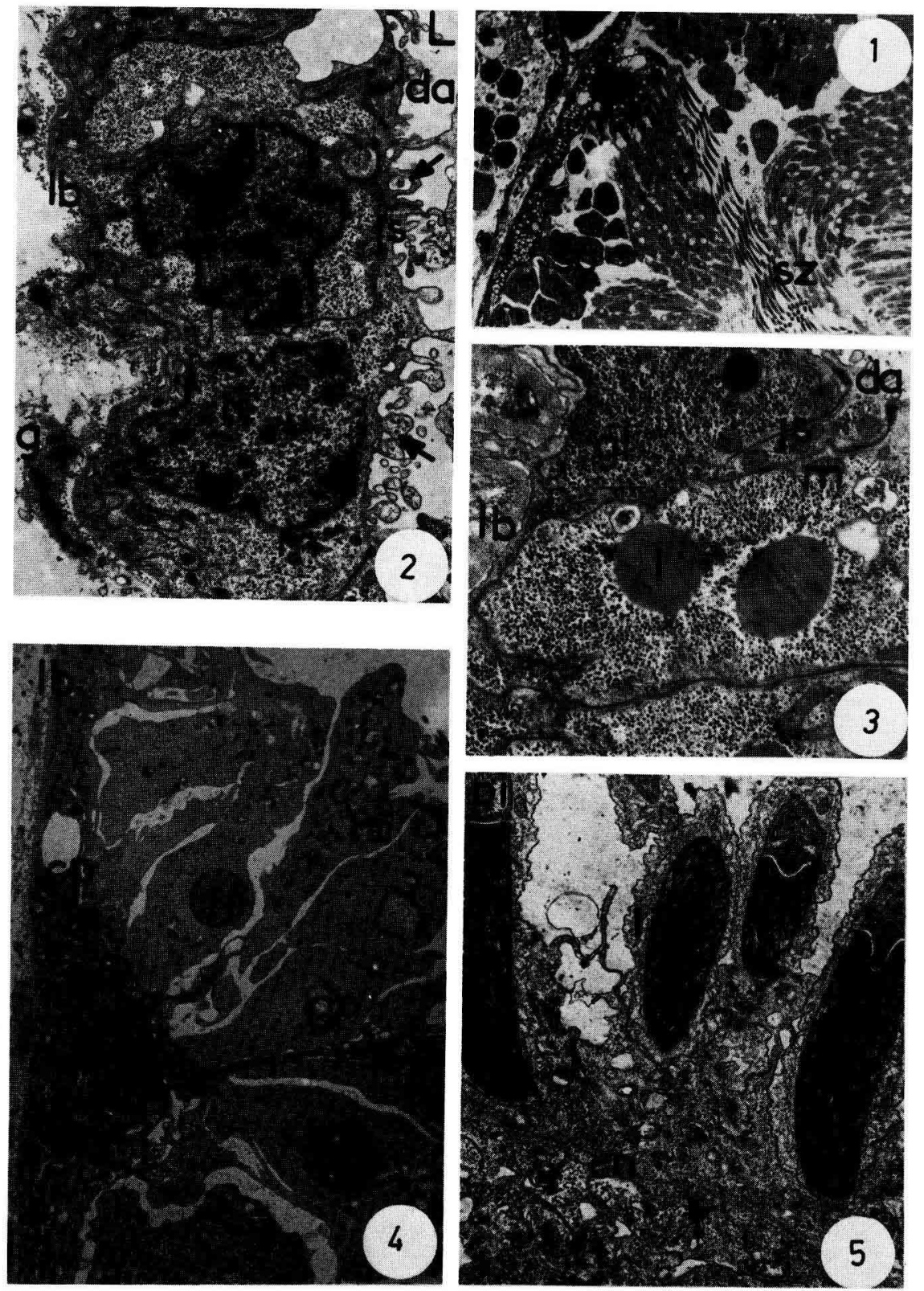

Gonade d'un Escargot élevé 39 jours en $\mathrm{J} L$ 
Au niveau ultrastructural, la lame basale apparaît souvent plissée, sans doute en raison du faible remplissage des acini : par endroits, aucune cellule ne lui est plus accolée. Par rapport aux Escargots élevés en $\mathrm{JL}$, quelques différences existent dans les cellules du col : le noyau, plus sombre, renferme de gros blocs hétérochromatiniens; les villosités sont moins nombreuses et moins développées (fig. 7), les mitochondries à matrice sombre sont très allongées et volumineuses. Dans la région distale, les cellules nourricières sont plus hautes et plus développées, aspect qui peut être corrélé à l'état d'avancement moins prononcé de la gamétogenèse mâle. Beaucoup possèdent un noyau volumineux, plus ou moins repoussé vers l'intérieur de la lumière. Nous n'observons pas d'anomalies dans la différenciation des cellules germinales: dans les ovocytes, préméiose, prévitellogenèse, vitellogenèse $\mathrm{I}$ et II se déroulent normalement. Pour la lignée mâle, les spermatocytes au stade zygotène abondent (fig. 8) ; groupés en bouquets autour des prolongements de cellules nourricières, ils présentent les complexes tripartites caractéristiques; leur cytoplasme renferme de très nombreuses mitochondries ainsi qu'un appareil de Golgi bien développé, localisé à la base du pédoncule par lequel ils s'accrochent aux cellules nourricières. Dans les spermatides rondes (fig. 8), la condensation de la chromatine et l'accumulation de matériel dense à deux pôles opposés de l'enveloppe nucléaire s'effectuent normalement. Quelques figures de dégénérescence (éclaircissement des noyaux, disparition de l'euchromatine) sont visibles dans des cellules nourricières ainsi que dans des cellules mâles ; de même, certains ovocytes en fin de vitellogenèse se nécrosent, mais ces phénomènes affectent un nombre restreint de cellules.

\section{2) Prélèvement P2.}

a) Escargots placés en JL. - 2 cas sont observés : dans 3 gonades sur 5 , les stades terminaux de la spermatogenèse, spermatides et spermatozoïdes sont extrêmement abondants et les cellules de la paroi distale sont très aplaties. Dans les 2 autres gonades, les cellules nourricières sont très hautes, très riches en lipides, elles envahissent la majeure partie de la lumière acineuse dans laquelle ne sont visibles que de rares spermatogonies et spermatocytes I. Ces observations suggèrent qu'il y a eu évacuation du sperme et que l'ovotestis est dans une phase de repos précédant une future vague gamétogénétique.

En ultrastructure, la lame basale des tubules vidés de leurs spermatozoïdes est plissée et dépourvue de cellules sur de grandes surfaces (fig. 9). Des débris cellulaires, fragments de membranes, gouttelettes lipidiques, noyaux nécrosés... encombrent la lumière. Par contre, dans les autres acini, la lame basale n'est pas ou peu plissée (fig. 10) ; elle supporte une ou parfois deux assises de cellules non germinales (fig. 10). L'assise la plus externe est alors constituée de cellules allongées parallèlement au bord de l'acinus, à noyau ovale (fig. 10) ou à contour irrégulier (fig. 11), à cytoplasme renfermant un appareil de Golgi bien développé, quelques lysosomes et des globules lipidiques. Face à la lame basale, ces cellules émettent de longs pseudopodes qui s'enchevêtrent avec ceux des cellules voisines. L'assise la plus interne est formée de cellules nourricières volumineuses, dont le cytoplasme a des caractères similaires (abondance de lipides, présence de lysosomes mais en beaucoup plus grand nombrel mais dont le noyau est très 

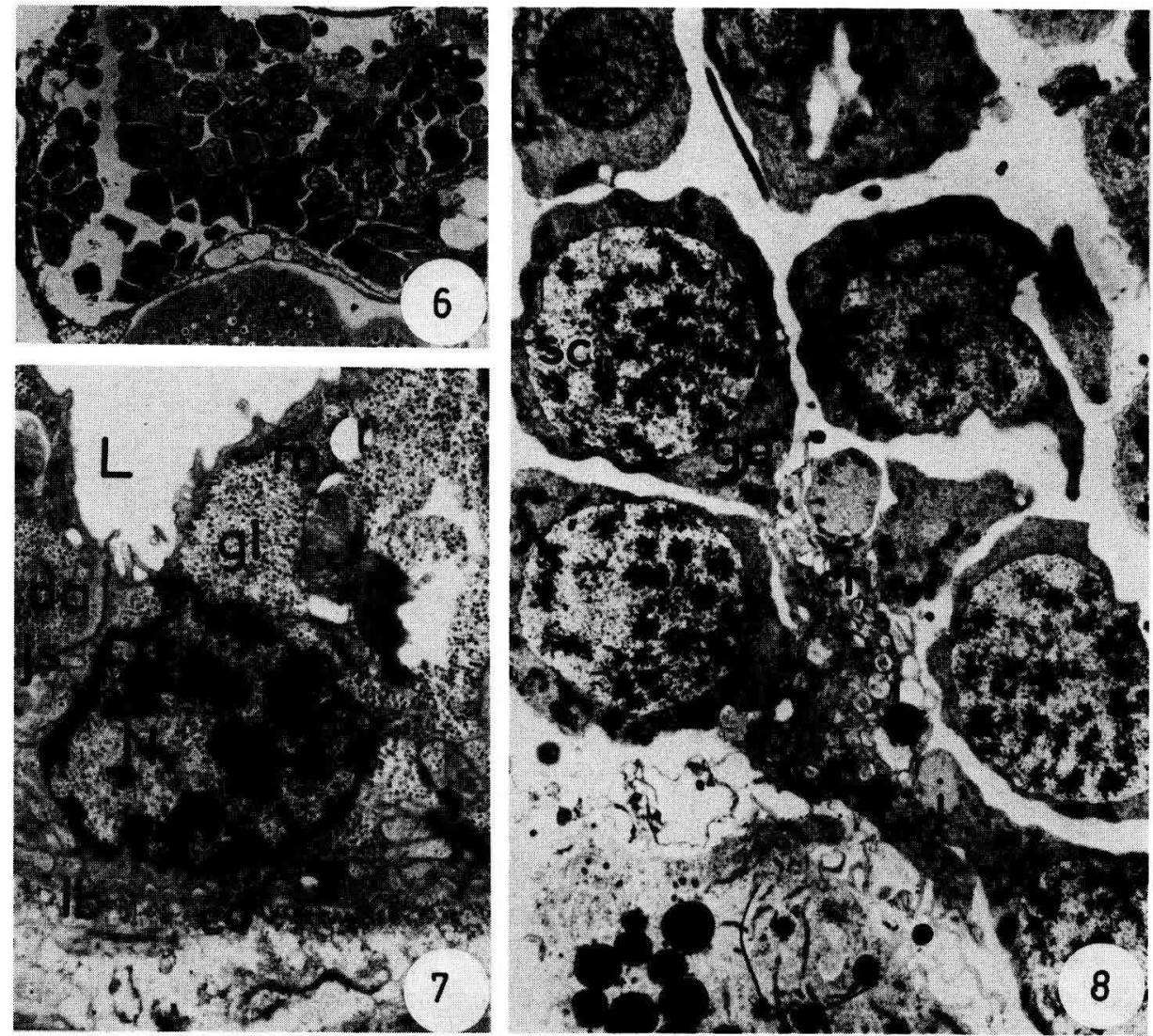

Gonade d'Escargot placé 39 jours en JC

FIG. 6. - Les acini renferment principalement des spermatogonies, des spermatocytes et quelques spermatides rondes. On remarque des figures de dégénérescence $(\times 300)$.

FIG. 7. - Cellule du col montrant un noyau riche en hétérochromatine, peu de villosités apicales et une trame de collagène périacineuse abondante $(\times 6300)$.

FIG. 8. - Bouquet de spermatocytes accrochés aux prolongements d'une cellule nourricière $(\times 3600)$.

cn : cellule nourricière ; co : collagène ; da : desmosome apical ; go : appareil de Golgi ; gl : glycogène $; \mathrm{j}$ : jonction entre $\mathrm{cn}$ et $\mathrm{sc} ;$ js : jonction septée $; \mathrm{L}:$ lumière $; \mathrm{I}:$ lipides $;$ lb : lame basale ; $\mathrm{m}$ : mitochondries ; $\mathbf{N}$ : noyau ; sc : spermatocytes ; sg : spermatogonies ; st : spermatides ; astérisques : figures de dégénérescence; flèches : accumulation de matériel dense contre l'enveloppe nucléaire d'une spermatide. 

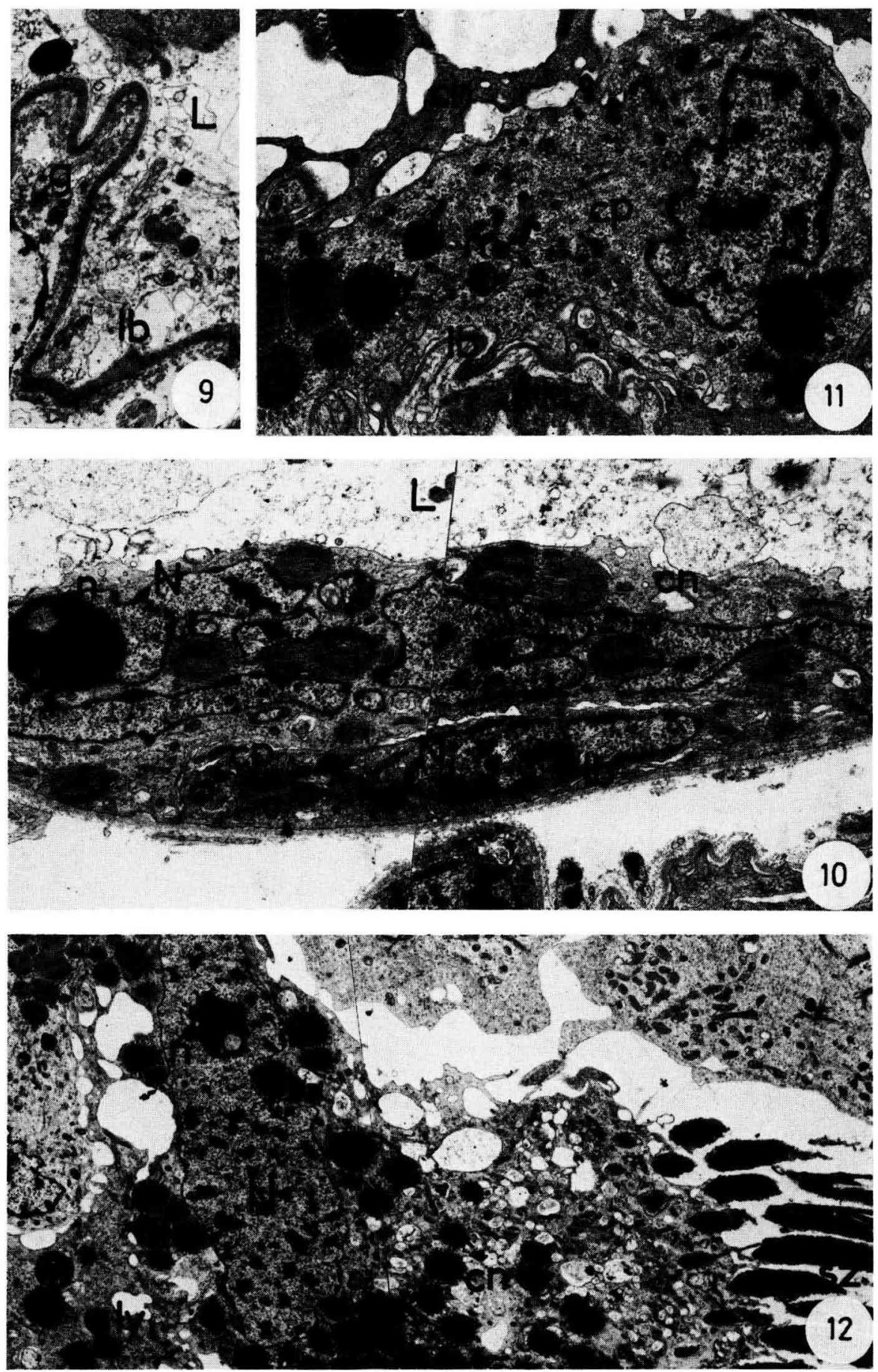

Gonade d'Escargot placé 69 jours en photophase longue (JL) 
différent ; repoussé vers la lumière, hypertrophié, il renferme une hétérochromatine dispersée en fines mottes et un nucléole réticulé lorsqu'un faisceau de spermatozoïdes est fiché dans le cytoplasme (fig. 12). Si au contraire la cellule nourricière ne supporte plus de cellules mâles, le noyau très allongé a un contour tourmenté, il s'étire en longues digitations et possède également un nucléole réticulé (fig. 10).

b) Escargots conservés en JC. - Spermatogonies et spermatocytes sont plus nombreux qu'en JL. Par rapport au prélèvement P1, les stades spermatides et spermatozoïdes sont atteints dans tous les cas, mais à côté de tubules bien remplis beaucoup apparaissent relativement vides. Les cellules de la paroi distale sont d'autant plus aplaties que la spermatogenèse est avancée et active. Dans les acini renfermant peu de cellules mâles, les cellules nourricières comblent presque totalement la lumière. Les figures de nécrose de cellules nourricières et germinales sont plus abondantes que dans tous les autres lots étudiés, du matériel en dégénérescence obstrue la lumière. Des cellules mâles tétranucléées sont observées, indiquant l'absence de cytodiérèse au cours de certaines divisions.

En microscopie électronique, les cellules de la paroi (fig. 13) et les cellules nourricières (fig. 14) ont sensiblement le même aspect qu'en JL. Les cellules germinales mâles, parvenues au terme de leur différenciation, semblent tout à fait normales.

\section{Analyse quantitative.}

Les données quantitatives sont regroupées dans le tableau $\mathrm{I}$.

1) Indices gonadiques. - La comparaison des indices ne fait pas apparaître de différences significatives entre les animaux placés en JL et en JC.

2) Teneur en protéines de la gonade. - Au moment du prélèvement $P 1$, alors que les gonades des animaux en JL et en JC ont le même poids moyen, la teneur en protéines est plus faible chez les premiers. La proportion relative de protéines diffère donc, ce qui implique une répartition différente des constituants non protéiques (sucres, lipides... des cellules nourricières et pariétales, réserves calciques ou lipidiques des ovocytes par exemple). En P2, la teneur en protéines a fortement augmenté aussi bien pour les $\mathrm{JC}$ que pour les $\mathrm{JL}$ et les valeurs obtenues sont très proches, confirmant les observations histologiques qui mettent en évidence les mêmes types cellulaires dans les deux conditions expérimentales.

FIG. 9. - Dans un tube gonadique ayant évacué ses spermatozoïdes, la lame basale est très plissée et la humière est occupée par des débris de clasmatose des cellules nourricières $(\times 4200)$.

FIG. 10. - Epithélium de la zone distale d'un acinus après spermiation avec une cellule pariétale allongée entre la lame basale et une cellule nourricière à noyau fortement découpé ( $\times 5500)$.

FIG. 11. - Cellule pariétale épousant intimement les contours de la lame basale $(\times 6800)$.

FIG. 12. - Juste avant la spermiation, les spermatozoïdes sont fichés dans le cytoplasme encombré de réserves d'une cellule nourricière à noyau polyploïde ( $\times 2400)$.

$\mathrm{cn}$ : cellule nourricière ; $\mathrm{cp}$ : cellule pariétale ; $\mathrm{g}:$ tissu glial ; $\mathrm{L}:$ lumière ; $\mathrm{l}$ : lipides ; lb : lame basale ; Iy : lysosomes ; $\mathrm{m}$ : mitochondries ; mu : muscle ; $\mathrm{N}$ : noyau ; $\mathbf{n}$ : nucléole ; sz : spermatozoïde. 


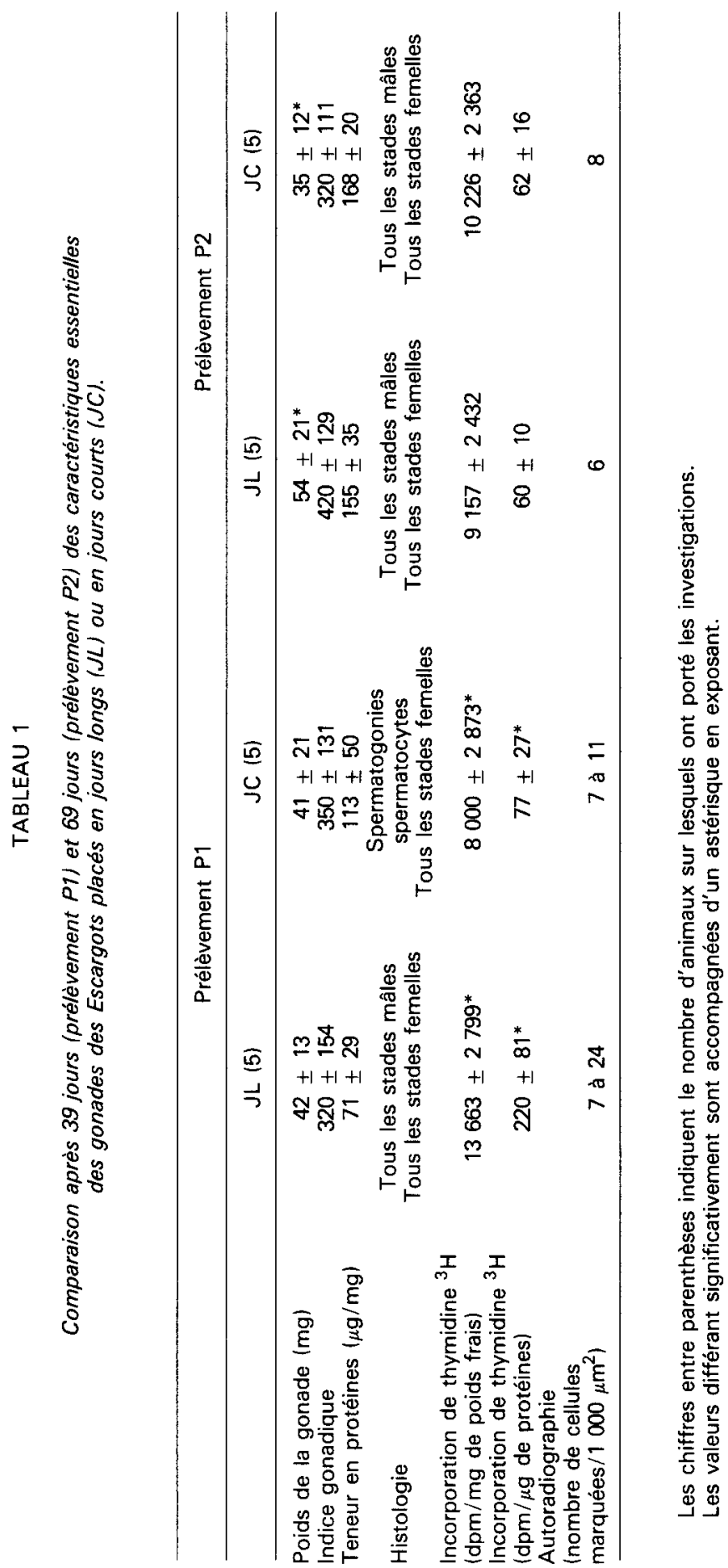


3) Evaluation de la synthèse d'ADN gonadique. - Pour P1, l'incorporation de thymidine tritiée est significativement plus importante en JL qu'en JC. La multiplication cellulaire est donc beaucoup plus intense en $\mathrm{JL}(\times 2$ ou 3 selon qu'on exprime la radioactivité en fonction du poids de la gonade ou de la quantité de protéines). Après 69 jours, le taux d'incorporation a diminué fortement chez les Escargots placés en JL, ce qui s'explique aisément car les animaux sont en fin de spermatogenèse ; il a varié plus faiblement chez les autres, la multiplication se poursuivant à peu près au même rythme.

4) Autoradiographie. -5 heures après l'injection de thymidine tritiée, les cellules marquées sont essentiellement des spermatogonies et quelques spermatocytes. Pour $\mathrm{P} 1$, elles constituent souvent des groupes isogéniques en $\mathrm{JL}$ alors qu'en JC, elles sont plutôt éparses dans les acini. Pour des surfaces de coupes équivalentes, le nombre de cellules ayant incorporé la thymidine est plus élevé en $\mathrm{JL}$. En P2, le nombre de cellules marquées et leur répartition sont très semblables dans les deux groupes d'animaux (fig. 15 et 16).

Par ailleurs, quelques noyaux de cellules nourricières sont fortement radioactifs en particulier en P1 chez les Escargots élevés en JC : cette observation prouve qu'il y a replication de l'ADN et polyploïdie de ces cellules dont le noyau s'accroît considérablement au cours du cycle spermatogénétique.

\section{Discussion - Conclusion.}

Les résultats conjugués de notre étude anatomique, cytologique, biochimique et autoradiographique démontrent que des Escargots placés à des photophases différentes après une hibernation de 4 mois ne réagissent pas de la même façon. Après 6 semaines en $\mathrm{JL}$, la multiplication spermatogoniale est active et de nombreuses cellules mâles sont parvenues au terme de la spermiogenèse. Par contre en JC, la synthèse d'ADN est plus faible et les cellules mâles dans leur majorité sont encore aux étapes précoces de la spermatogenèse. Ces observations rejoignent celles d'Henderson et Pelluet (1960) qui montrent qu'un éclairement continu stimule la spermiogenèse de Deroceras reticulatum, de Sokolove et al. (1984) qui observent en JL une augmentation de la multiplication spermatogoniale de Limax maximus. Chez Helix aspersa après 10 semaines, les différences s'estompent : les animaux placés en JL sont en fin de spermiogenèse et chez les animaux de JC, des spermatozoïdes, bien que moins abondants, se sont différenciés. Contrairement aux basses températures (Gomot et al., 1986), les photophases courtes ne provoquent pas un blocage de la lignée mâle à un stade précis ; elles n'entraînent pas non plus l'apparition d'une forte proportion d'anomalies dans la différenciation cytosexuelle. Mais d'une part elles ralentissent le déroulement de la spermatogenèse, d'autre part elles abaissent le taux de multiplication cellulaire, ce qui a pour répercussion une densité finale de spermatozoïdes beaucoup plus faible que chez les animaux de JL. Ces résultats rejoignent ceux de Hunter et Stone (1986) qui, chez Cepaea nemoralis, notent un retard dans le début de la ponte et un moins grand nombre d'œufs pondus chez les animaux de JC. 

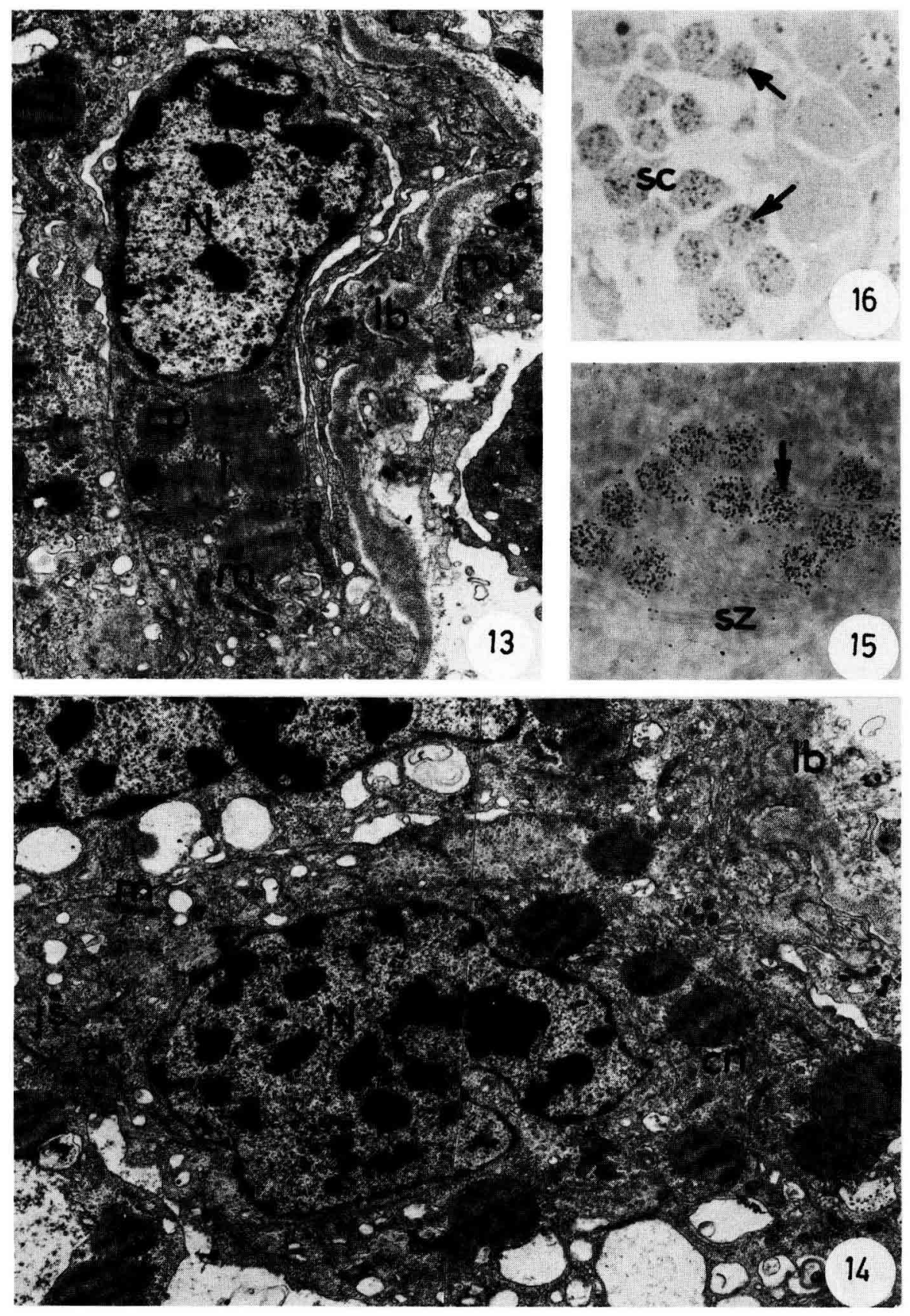

Gonade d'un Escargot placé 69 jours en photophase courte (JC) 
En ce qui concerne la lignée femelle, tous les stades de son évolution sont observés dans les deux conditions expérimentales analysées, mais sans doute les proportions de chaque catégorie d'ovocytes diffèrent-elles, comme le suggèrent les variations de la teneur en protéines. Des comptages de chaque classe ovocytaire devront être effectués pour préciser le comportement des cellules femelles.

Au niveau des cellules de la paroi, cellules du col et cellules nourricières, des différences sont notées entre animaux de $\mathrm{JC}$ et de $\mathrm{JL}$, en particulier au moment de $\mathrm{P} 1$; elles semblent liées à un asynchronisme de fonctionnement des gonades plutôt qu'à des perturbations physiologiques. En effet, les cellules nourricières par exemple présentent un cycle en relation avec l'état de différenciation de la lignée mâle, comme chez Biomphalaria glabrata (De Jong-Brink et al., 1977). Les dissemblances dans leur taille, leur position, leur forme... traduisent un décalage dans leur évolution.

Dans nos conditions expérimentales (températures alternées de 17 et $12^{\circ} \mathrm{C}$ ), les courtes photophases entraînent une absence de reproduction, alors que chez un autre Hélicidé, à des températures alternées de 28 et $23^{\circ} \mathrm{C}$, elles n'inhibent pas la ponte (Hunter et Stone, 1986). D'autres séries expérimentales sont donc nécessaires pour définir lequel des deux facteurs, température ou lumière, joue chez Helix aspersa le rôle de facteur critique.

Quoiqu'il en soit, nos observations mettent en évidence une action primaire de la lumière sur la gamétogenèse qui, dans le cas des $\mathrm{JC}$, n'exerce pas une stimulation suffisante des centres neuro-endocrines qui contrôlent la reproduction.

Recu en octobre 1986.

Accepté en décembre 1986.

Remerciements. - Les auteurs adressent leurs remerciements au personnel du laboratoire et plus particulièrement à Brigitte Jolibois pour la mise en page du manuscrit et à Félix Sanchez pour la réalisation des planches photographiques.

FIG. 13. - Cellule pariétale émettant de nombreux prolongements contre la lame basale et tout à fait comparable à celle de la Fig. $11(\times 6800)$.

FIG. 14. - Cellule nourricière d'une zone peu activée de l'épithélium acineux avec un noyau lobé et un cytoplasme riche en globules lipidiques. On remarque sa liaison étroite avec une cellule nourricière voisine $(\times 6800)$

cn : cellule nourricière ; $c \rho$ : cellule pariétale; $d$ : desmosome; $g$ : cellule gliale ; js : jonction septée ; 1 : lipides ; $\mathrm{lb}$ : lame basale ; $\mathrm{m}$ : mitochondries ; mu : fibres musculaires ; $\mathrm{N}: \mathrm{noyau}$; $\mathrm{n}$ : nucléole.

FIG. 15. - Bouquet de cellules mâles marquées dans la gonade d'un Escargot élevé 69 jours en $J L(\times 680)$.

FIG. 16. - Spermatogonies marquées dans la gonade d'un Escargot élevé 69 jours en JC $(\times 680)$.

Sz : spermatozoïdes ; flèches : marquage des cellules mâles. 


\section{Références}

BOHLKEN S., JOOSSE J., 1982. The effect of photoperiod on female reproductive activity and growth of the freshwater pulmonate snail Lymnaea stagnalis kept under laboratory breeding conditions. Int. J. Invert. Reprod. Dev., 4, 213-222.

BONNEFOY-CLAUDET R., DERAY A., 1984. Influence de la durée d'hibernation sur l'activité reproductrice de l'Escargot Helix aspersa Müller. C. R. Soc. Biol., 178, 442-449.

COURTOT A. M., GOMOT L., 1982. Les stades de l'organogenèse et la cytodifférenciation de la glande à albumen de I'Escargot Helix aspersa Müller. Reprod. Nutr. Dév., 22, 661-680.

DE JONG-BRINK M., BOER H. H., HOMMES T. G., KODDE A., 1977. Spermatogenesis and the role of Sertoli cells in the freshwater snail Biomphalaria glabrata. Cell Tiss. Res., 181, 37-58.

ENEEE J., BONNEFOY-CLAUDET R., GOMOT L., 1982. Effet de la photopériode artificielle sur la reproduction de l'Escargot Helix aspersa Müller. C. R. Acad. Sci. Paris, 294, 357-360.

GOMOT P., GOMOT L., 1985. Action de la photopériode sur la multiplication spermatogoniale et la reproduction de l'Escargot Helix aspersa. Bull. Soc. Zool. Fr., 110, 445-459.

GOMOT P., GRIFFOND B., GOMOT L., 1986. Effets de la température sur la spermatogenèse d'Escargots Helix aspersa maintenus en repos artificiel. C. R. Acad. Sci. Paris, 302, 27-32.

GRIFFOND B., BOLZONI-SUNGUR D., 1986. Stages of oogenesis in the snail, Helix aspersa : cytological, cytochemical and ultrastructural studies. Reprod. Nutr. Dév., 26, 461-474.

HENDERSON N. E., PELLUET D., 1960. The effect of visible light on the ovotestis of the slug Deroceras reticulatum (Müller). Can. J. Zool., 38, 173-178.

HUNTER R. D., STONE L. M., 1986. The effect of artificial photoperiod on growth and reproduction in the land snail Cepaea nemoralis. Int. J. Invert. Reprod. Dev., 9, 339-344.

JOOSSE J., 1984. Photoperiodicity, rhythmicity and endocrinology of reproduction in the snail Lymnaea stagnalis, 204-220. In Photoperiodic regulation of insect and molluscan hormones, Pitman, London (Ciba Foundation Symposium 104).

LOWRY O. H., ROSEBROUGH N. J., FARR A. L., RANDALL R. J., 1951. Protein measurement with the folin phenol reagent. J. biol. Chem., 193, 265-275.

REYNOLDS E. S., 1963. The use lead citrate at high $\mathrm{pH}$ as an electron opaque stain in electron microscopy. J. Cell Biol., 17, 208-212.

SIEGEL S., 1956. The case of two independent samples in non parametric statistics. International Student (Ed) McGraw Hill International Book Company, Auckland, p. 116.

SOKOLOVE P. G., McCRONE E. J., 1978. Reproductive maturation in the slug Limax maximus and the effects of artificial photoperiod. J. comp. Physiol., 125, 317-325.

SOKOLOVE P. G., MELROSE G. R., GORDON T. M., O'NEILL M. C., 1983. Stimulation of spermatogonial DNA synthesis in slug gonad by a factor released from cerebral ganglia under the influence of long days. Gen. comp. Endocrinol., 50, 95-104.

SOKOLOVE P. G., MCCRONE E. J., VAN MINNEN J., DUNCAN W. C., 1984. Reproductive endocrinology and photoperiodism in a terrestrial slug, 189-203. In Photoperiodic regulation of insect and molluscan hormones, Pitman, London (Ciba Foundation Symposium 104).

STEPHENS G. J., STEPHENS G. C., 1966. Photoperiodic stimulation of egg laying in the land snail Helix aspersa. Nature, 212, 1582.

TARDY J., 1982. Action des facteurs externes sur la sexualité des Mollusques Gastéropodes aquatiques. Bull. Soc. Zool. Fr., 107, 53-70.

TRUMP B. F., SMUCKLER E. A., BENDITT E. P., 1961. A method for staining epoxy sections for light microscopy. J. U/trastruct. Res., 5, 343-345. 